\title{
On Some Groups of Automorphisms of von Neumann Algebras with Cyclic and Separating Vector
}

\author{
A. Z. JADCZYK \\ Institute of Theoretical Physics, University of Wrocław
}

Received January 6, 1969

\begin{abstract}
Let $\mathfrak{A}$ be a von Neumann algebra with the vector $\Omega$ cyclic and separating for $\mathfrak{A}$. Let $\mathfrak{V}_{G}$ be a group of unitary operators under which both $\Omega$ and $\mathfrak{A}$ are invariant. Let $\mathfrak{B}$ (resp. $\mathfrak{R}^{\prime}$ ) be the fixed point algebra in $\mathfrak{A}$ (resp. in $\mathfrak{H}^{\prime}$ ). Let $F_{0}$ be an orthogonal projection onto the subspace of all vectors invariant under $\mathfrak{V}_{G}$. It is shown that $\mathfrak{R}=\left(\mathfrak{A} \cup\left\{F_{0}\right\}\right)^{\prime \prime}$ and that the irreducibility of $\mathfrak{R}$ implies that $F_{0}$ is one-dimentional. Other consequences of the Theorem of Kovícs and Szücs are also derived. In sec. 3 . the spectrum properties of the group $\mathfrak{V}_{G}$ are studied. It is proved that the point spectrum of $\mathfrak{V}_{G}$ is symmetric and that it is a group provided $\mathfrak{R}$ is irreducible. In this case there exists a homomorphism $\chi \rightarrow \hat{\chi}$ (resp. $\chi \rightarrow \check{\chi}$ ) of the point spectrum of $\mathfrak{V}_{G}$ into the group of unitary operators in $\mathfrak{A}$ (resp. in $\mathfrak{A}^{\prime}$ ) uniquely (up to the phase) defined by $\hat{\chi} V_{g}=\chi(g) V_{g} \hat{\chi}$ (resp. the same for $\check{\chi}$ ). In sec. 4 . the application of the foregoing results to the KMS-Algebra is given.
\end{abstract}

\section{Introduction}

It has been shown in [1] that the state of thermal equilibrium of an infinite system is mathematically described by the state $\omega$ (over the $C^{*}$. algebra of observables $\mathfrak{A}_{0}$ ) satisfying the KMS boundary conditions. Let $\left(\pi, \mathscr{H}_{\pi}\right)$ be a canonical representation defined by $\omega$, and let $\Omega$ be the vector representing the state $\omega$ in $\mathscr{H}_{\pi}$. Finally, let $\mathfrak{A}=\pi\left(\mathfrak{A}_{0}\right)^{\prime \prime}$. It has been exhibited in [1] that there is a peculiar symmetry between $\mathfrak{A}$ and $\mathfrak{A}^{\prime}$. In particular, the vector $\Omega$ invariant under time and space translations is cyclic for both, $\mathfrak{A}$ and $\mathfrak{A}^{\prime}$. A similar situation arises when one deals with internal symmetries in the framework of Algebraic Quantum Field Theory. The goal of our paper is to study a general situation, when there is given a von Neumann algebra $\mathfrak{A}$, the group $A \rightarrow V_{g} A V_{g}^{-1}$ of automorphisms of $\mathfrak{A}$ and the vector $\Omega$ invariant under all $V_{g}$ and cyclic for $\mathfrak{A}$ and $\mathfrak{A}^{\prime}$. A general discussion of such a situation is given in Section 2 . Our tool, in this section, is the theorem of Kovács and SzÜCS (see [4] and [4a, Theorem 1]). It is shown that the irreducibility of $\mathscr{A} \cup\left\{V_{g}\right\}$ implies that $\Omega$ is the unique vector invariant under all $V_{g}$. We also show that $\left(\mathfrak{A} \cup\left\{V_{g}\right\}\right)^{\prime \prime}=\left(\mathfrak{A} \cup\left\{F_{0}\right\}\right)^{\prime \prime}$, where $F_{0}$ is a projection onto the subspace 
of all vectors invariant under $\left\{V_{g}\right\}$. In Section 3 we deal with the spectrum properties of the group $\mathfrak{V}_{G}$. The results of this section are closely related to those obtained by D. W. RoBINson [3]. In particular we prove that the point spectrum of $\mathfrak{V}_{G}$ is symmetric and it is a group provided $\mathfrak{V}_{G}$ commutes with another group $\mathfrak{V}_{G_{1}}$ such that $\mathfrak{V}_{G_{1}} \cup \mathfrak{A}$ is irreducible. The Theorem 3.5. is the main result of this section. Finally, in Section 4. we apply the results of the foregoing sections to the case of the KMS-Algebra. In particular, for each discrete eigenvalue of the "momentum" $p$ we construct two unitary operators $\hat{p} \in \mathfrak{A}$ and $\check{p} \in \mathfrak{A}^{\prime}$ such that

$$
\begin{aligned}
\hat{p} \hat{q} & =(p+q)^{\curlywedge}, & \check{p} \check{q} & =(p+q)^{`}, \\
\hat{p} V_{x} & =e^{i p x} V_{x} \hat{p}, & \check{p} V_{x} & =e^{i p x} V_{x} \check{p} .
\end{aligned}
$$

It is shown that for each such $p$, the vector $\psi_{p}=\hat{p} \Omega$ also describes the state of thermal equilibrium, with the same temperature as $\Omega$.

\section{General Discussion}

In this section we are concerned with some properties of groups of automorphisms of a von Neumann algebra with a cyclic and separating vector. The following results are a simple application of the theorem of KovÁCS and Szücs (see [4] and (4a, Theorem 1]).

2.1. Notation. Let $\mathfrak{A}$ be a von Neumann algebra acting on a Hilbert space $\mathscr{H}$, with commutant $\mathfrak{A}$ and the center $\mathcal{B}$. Let $G$ be a group and $g \rightarrow V_{g}$ be a unitary representation of $G$ on $\mathscr{H}$ such that $A(g)=V_{g} A V_{g}^{-1}$ is in $\mathfrak{A}$ for each $A$ in $\mathfrak{A}$. Let $F_{0}$ be a projection onto the subspace of all $\psi$ in $\mathscr{H}$, invariant under all $V_{g}$. Denoting by $\mathfrak{V}_{G}$ an image of $G$ under the mapping $g \rightarrow V_{g}$ let $\mathfrak{R}$ be a von Newmann algebra generated by $\mathfrak{A}$ and $\mathfrak{Y}_{G}$, and let $\mathfrak{B}$ be a fixed point algebra in $\mathfrak{A}$ i.e. $\mathfrak{B}=\mathfrak{A} \cap \mathfrak{V}_{G}^{\prime}$. We remark that $\mathfrak{H}^{\prime}$ is now also invariant under $G$, with a fixed point algebra $\mathfrak{R}^{\prime}$. Finally, let $\mathscr{M}$ be a Godement mean over $G$ (see e.g. [4a]). This notation will be fixed throughout this section.

2.2. Lemma. Assume there exists a unit vector $\Omega$ which is cyclic and separating for $\mathfrak{A}$ and such that $V_{g} \Omega=\Omega$ for all $g$ in $G$. Then

I. There exist two unique normal G-invariant projection maps $\Phi$ and $\Phi^{\prime}$ from $\mathfrak{A}$ onto $\mathfrak{B}$ and from $\mathfrak{A}^{\prime}$ onto $\mathfrak{R}^{\prime}$ respectively. $\Phi$ and $\Phi^{\prime}$ are positive and faithful. $\Phi(A)$ (resp. $\Phi^{\prime}(A)$ ) can be defined (equivalently) as

i) the unique element of $\mathfrak{A} \cap \operatorname{conv}\{A(\hat{g})\}^{-}\left(\operatorname{resp} \cdot \mathfrak{A}^{\prime} \cap \operatorname{conv}\{A(\hat{g})\}^{-}\right)$

ii) the unique element of $\mathfrak{A}$ (resp. $\left.\mathfrak{A}^{\prime}\right)$ such that $\Phi(A) F_{0}=F_{0} A F_{0}$ (resp. $\left.\Phi^{\prime}(A) F_{0}=F_{0} A F_{0}\right)$,

iii) the unique operator on $\mathscr{H}$ satisfying $(\varphi, \Phi(A) \psi)=\mathscr{M}\{(\varphi, A(\hat{g}) \psi)\}$ for all $\varphi, \psi \in \mathscr{H}$ (resp. the same for $\left.\Phi^{\prime}(A)\right)$,

II. A normal linear from $\omega$ on $\mathfrak{A}$ (resp. $\left.\mathfrak{A}^{\prime}\right)$ is $G$-invariant if and only if $\omega=(\omega \mid \mathfrak{B}) \circ \Phi\left(\right.$ resp. $\left.\omega=\left(\omega \mid \mathfrak{R}^{\prime}\right) \circ \Phi^{\prime}\right)$. 
III. With $A$ in $\mathfrak{A}\left(\right.$ resp. $\left.\mathfrak{A}^{\prime}\right)$ the following are equivalent

$$
\begin{aligned}
\text { (p) } & F_{0} A=A F_{0}, \\
(p p) & \left.A \in \mathfrak{B} \quad \text { (resp. } A \in \mathfrak{R}^{\prime}\right)
\end{aligned}
$$

IV. $F_{0}=[\mathcal{B} \Omega]=\left[\mathfrak{R}^{\prime} \Omega\right]$.

Proof. I, i)-iii) are an immediate consequence of [4a, Theorem 1]. To prove III suppose $F_{0} A=A F_{0}$ where $A$ is in 2 . Then $A F_{0}=F_{0} A F_{0}$ and we find that, by ii), $A=\Phi(A) \in \mathfrak{B}$. The case of $A \in \mathfrak{X}^{\prime}$ is handled in exactly the same way. Implication $(p p) \rightarrow(p)$ is obvious. Finally we will prove IV. It is clear that $F_{0} \geqq[\mathfrak{B} \Omega]$ so it is sufficient to check that $\mathfrak{B} \Omega$ is dense in $F_{0} \mathscr{H}$. Given $\psi \in F_{0} \mathscr{H}$ we can choose a sequence $A_{n} \in \mathfrak{A}$ such that $A_{n} \Omega \rightarrow \psi$. But then

$$
\psi=F_{0} \psi=\lim F_{0} A_{n} \Omega=\lim \Phi\left(A_{n}\right) \Omega
$$

and the proof is complete.

As a corollary of the foregoing lemma we have

2.3. Corollary. Let $G$ be as in Lemma 2.2. Then

i) $F_{0} \mathfrak{R} F_{0}=\mathfrak{B} F_{0} \cong \mathfrak{B}$

$$
F_{0} \mathfrak{B}^{\prime} F_{0}=\mathfrak{R}^{\prime} F_{0} \cong \mathfrak{R}^{\prime}
$$

and $\mathfrak{B}$ is abelian (resp. finite, semi-finite, properly infinite, purely infinite) if and only if $\mathfrak{R}^{\prime}$ is abelian (resp. finite, semi-finite, properly infinite, purely infinite). Moreover, if $\mathfrak{B}$ is abelian then $\mathfrak{B} \cong \mathfrak{B} F_{0}=\mathfrak{R}^{\prime} F_{0} \cong \mathfrak{R}^{\prime}$.

ii) If $G_{1}$ is another group satisfying assumptions of Lemma 2.2 then one has an equivalence of

$$
\begin{aligned}
(p) & \mathfrak{B}_{1} \subset \mathfrak{B} \\
(p p) & \Re_{1} \supset \mathfrak{R} \\
(p p p) & F_{01} \leqq F_{0}
\end{aligned}
$$

In particular, if for all $A, B \in \mathfrak{A}$ and $\varphi, \psi \in F_{01} \mathscr{H}$

$$
\mathscr{M}\left\{\left(\varphi,\left[V_{\hat{g}} A V_{\hat{g}}^{-1}, B\right] \psi\right)\right\}=0, \quad g \in G_{1}
$$

and $\mathcal{B} \subset \mathfrak{B}$, then all $(p)-(p p p)$ are satisfied.

Proof. The first statement follows by [4a, Corollary 2]. Now, by [7, Chapter III, $\S 2$, Prop. 3], if $\mathfrak{B}$ is abelian etc. so does $\mathfrak{R}^{\prime}$. On the other hand, if $\mathfrak{B}$ is abelian, then $\mathfrak{B} F_{0}, \mathfrak{R}^{\prime} F_{0}$ and $\mathfrak{R}^{\prime}$ are abelian and therefore $\mathfrak{B} F_{0}=\left(\mathfrak{R} \cap \mathfrak{R}^{\prime}\right) F_{0}=\mathfrak{R}^{\prime} F_{0}$. Implications $(p) \rightarrow(p p p)$ and $(p p) \rightarrow(p p p)$ are an immediate consequence of Lemma 2.2, IV. On the other hand, if $A$ is in $\mathcal{B}_{1}$ then $F_{01} A \Omega$ and if $(p p p)$ holds then also $F_{0} A \Omega=A \Omega$ or $\Phi(A) \Omega=A \Omega$. Thus $A=\Phi(A) \in \mathfrak{Z}$. Implication $(p p p) \rightarrow(p p)$ is ob. tained in a much the same way. Finally, if $\mathfrak{B} \supset \mathcal{B}$ and $G_{1}$ satisfies the last assumption of the Corollary, then it follows from [4a, Lemma 2] that $\mathfrak{R}_{1}^{\prime} \subset \mathcal{B}$, and so $\mathfrak{R}_{1}^{\prime} \subset \mathcal{B} \subset \mathfrak{R}^{\prime}$. Q.E.D. 
Our next task is to show that the cyclicity of $\Omega$ for $\mathfrak{A}^{\prime}$ can in a sence replace the asymptotic abelianness. This can be also seen in the next section, where the spectrum properties are studied. The theorem given below should be compared with [5, Theorem 6]. (A similar statement, stated in a less general form has been proved by H. ARAKI and H. MiYata [8]).

2.4. Theorem. With notation and assumptions of Lemma 2.2. the following are equivalent.

i) The state $\omega(A)=(\Omega, A \Omega)$ is an extremal $G$-invariant state over $\mathfrak{A}$,

ii) $\mathfrak{R}^{\prime}=\{\lambda I\}$,

iii) $F_{0}$ is one-dimentional,

iv) $\mathfrak{B}=\{\lambda I\}$,

v) $\Phi(A)=(\Omega, A \Omega)$ for all $A$ in $\mathfrak{A}$,

vi) $\omega$ is weakly clustering $\mathscr{M}\{\omega(A(\hat{g}) B)\}=\omega(A) \omega(B)$ for all $A, B \in \mathfrak{A}$,

vii) $\omega$ satisfies the "stability" condition:

a) $\mathscr{M}\left\{\omega\left(B^{*} A(\hat{g}) B\right)\right\}=\omega\left(B^{*} B\right) \omega(A)$ for all $A, B \in \mathfrak{A}$,

b) $\mathscr{M}\{\omega(C A(\hat{g}) B)\}=\omega(C B) \omega(A)$ for all $A, B, C \in \mathfrak{A}$.

Proof. For implications i) $\Leftrightarrow$ ii) $\leftarrow$ iii) $\rightarrow$ iv) see for example [9], Theorem 4. Suppose now that $F_{0}=[\Omega]$. Then, by Lemma 2.2, IV, we have $A \Omega=\lambda \Omega$ for $A$ in $\mathfrak{B}$ and $A$ in $\mathfrak{R}^{\prime}$. Hence $\mathfrak{B}=\{\lambda I\}$ and $\mathfrak{R}^{\prime}=\{\lambda I\}$. It is evident that iv) implies $\mathrm{v}$ ) and if $\mathrm{v}$ ) holds then

$$
\begin{aligned}
\mathscr{M}\{\omega(C A(\hat{g}) B)\}=\mathscr{M}\{(\Omega, C A(\hat{g}) B \Omega)\} & =(\Omega, C \Phi(A) B \Omega) \\
& =(\Omega, A \Omega)(\Omega, C B \Omega) .
\end{aligned}
$$

But this means that it is sufficient to prove vi) $\rightarrow \mathrm{v}$ ). However, the latter is obvious if we notice that

$$
\mathscr{M}\{\omega(A(\hat{g}) B)\}=(\Omega, \Phi(A) B \Omega)
$$

and $\Omega$ is.separating for $\mathscr{A}$.

\section{Properties of the Spectrum}

It is of some interest that some group properties of the spectrum, typical for asymptotically abelian systems (see e.g. [10], Theorem 3a) appear also in the situation discussed in the preceding section. Our method of exhibiting these properties makes it possible to adapt the considerations of this section to the study of the spectrum of internal symmetries in the Algebraic Quantum Field Theory (see [11, 11a]).

3.1. Definition. Let $G \rightarrow \mathfrak{V}_{G}$ be a unitary representation of the group $G$ on a Hilbert space $\mathscr{H}$. Let $\hat{G}$ be the group of all (bounded) characters of the group $G$. With $\chi$ in $\hat{G}$ we say that $\chi$ is in a point spectrum of $\mathfrak{V}_{G}$ if there is $0 \neq \psi \in \mathscr{H}$ such that

10 Commun.math. Phys., Vol. 13

$$
V_{g} \psi=\chi(g) \psi \quad \text { for all } g \in G .
$$


Let $\sigma\left(\mathfrak{V}_{G}\right)$ be the set of all such characters. Let for each $\chi \in \hat{G}, F(\chi)$ be a projection onto the (closed) subspace of all $\psi$ in $\mathscr{H}$ satisfying (*). It is clear that $\chi \in \sigma\left(\mathfrak{V}_{G}\right)$ if and only if $F(\chi) \neq 0$, and by

$$
(\varphi, F(\chi) \psi)=\mathscr{M}\left\{\chi(\hat{g})^{-1}\left(\varphi, V_{\hat{g}} \psi\right)\right\} \equiv \mathscr{M}\left\{\chi(\hat{g})\left(\varphi, V_{\hat{g}}^{-1} \psi\right)\right\}
$$

if and only if the righ-hand side is non-zero for some $\varphi, \psi \in \mathscr{H}$.

3.2. Theorem. Let $\mathfrak{A}$ and $\left\{V_{g}\right\}$ be as in 2.1. Assume that $F_{0}$ has central carrier $I$ in $\mathfrak{R}$ (or equivalently, is cyclic for $\mathfrak{A}$ ), and $\mathfrak{V}_{G}$ commutes with some other $\mathfrak{V}_{G_{1}}$ such that $\mathfrak{V}_{G_{1}} \mathfrak{A} \mathfrak{V}_{G_{1}}^{*}=\mathfrak{A}$ and $\mathfrak{V}_{G_{1}}^{\prime} \cap \mathfrak{U}=\{\lambda I\}$. Then $\sigma\left(\mathfrak{V}_{G}\right)$ is a subgroup of the character group $\hat{G}$ of $G$.

Proof. We first observe that, due to the irreducibility of $\mathfrak{V}_{G_{1}} \cup \mathfrak{A}^{\prime}$, $F(\chi)$ is cyclic for $\mathfrak{V}_{G_{1}} \cup \mathfrak{A}^{\prime}$ for each $\chi$ in $\sigma\left(\mathfrak{V}_{G}\right)$. On the other hand $F(\chi) \mathscr{H}$ is an invariant subspace for $\mathfrak{V}_{G_{1}^{\prime}}$, and therefore must be cyclic for $\mathfrak{A}^{\prime}$ (we also have $\left.\mathfrak{V}_{G_{1}} \mathfrak{U}^{\prime} \mathfrak{V}_{G_{1}}^{*}=\mathfrak{A}^{\prime}\right)$. Now, let $\chi, \chi^{\prime} \in \sigma\left(\mathfrak{V}_{G^{\prime}}\right)$. For an arbitrary $\varphi, \psi \in \mathscr{H}, A \in \mathfrak{A}, B \in \mathfrak{U}^{\prime}$ we then have

$$
\begin{aligned}
\mathscr{M}\left\{\chi(\hat{g})^{-1}\left(F\left(\chi^{\prime}\right) \varphi, A(\hat{g}) B^{*} F_{0} \psi\right)\right\} & =\mathscr{M}\left\{\chi(\hat{g})^{-1}\left(F\left(\chi^{\prime}\right) \varphi, B^{*} V_{\hat{g}} A F_{0} \psi\right)\right\} \\
& =\left(B F\left(\chi^{\prime}\right) \varphi, F(\chi) A F_{0} \psi\right) .
\end{aligned}
$$

On the other hand

$$
\begin{aligned}
\mathscr{M}\left\{\chi(g)^{-1}\left(F\left(\chi^{\prime}\right) \varphi, A(\hat{g}) B^{*} F_{0} \psi\right)\right\} & =\mathscr{M}\left\{\chi^{-1} \chi^{\prime}(\hat{g})\left(F\left(\chi^{\prime}\right) \varphi, A V_{\hat{g}}^{*} B^{*} F_{0} \psi\right)\right\} \\
& =\left(F\left(\chi^{\prime}\right) \varphi, A F\left(\chi^{-1} \chi^{\prime}\right) B^{*} F_{0} \psi\right)
\end{aligned}
$$

so we have

$$
\left(B F\left(\chi^{\prime}\right) \varphi, F(\chi) A F_{0} \psi\right)=\left(F\left(\chi^{\prime}\right) \varphi, A F\left(\chi^{-1} \chi^{\prime}\right) B^{*} F_{0} \psi\right)
$$

for all $A \in \mathfrak{A}, B \in \mathfrak{A}^{\prime}$ and $\varphi, \psi$ in $\mathscr{H}$. However, by the hypothesis, $F\left(\chi^{\prime}\right)$ is cyclic for $\mathfrak{A}^{\prime}$ and $F_{0}$ is cyclic for $\mathfrak{A}$, so we conclude that the left-hand side is non-zero for some $A, B, \varphi, \psi$ and therefore $F\left(\chi^{-1} \chi^{\prime}\right) \neq 0$ or $\chi^{-1} \chi^{\prime} \in \sigma\left(\mathfrak{V}_{G^{\prime}}\right)$. It follows that with $\chi \in \sigma\left(\mathfrak{V}_{G}\right)$ also $\chi^{-1}$ is in $\sigma\left(\mathfrak{V}_{G}\right)$ and if $\chi$ and $\chi^{\prime}$ are in $\sigma\left(\mathfrak{V}_{G}\right)$ then $\chi \cdot \chi^{\prime}$ does also. Q.E.D.

By putting $\chi^{\prime}=1$ in the last equality we see that $F\left(\chi^{-1}\right) \neq 0$ once $F_{0}$ is cyclic for $\mathfrak{A}$ and $\mathfrak{A}^{\prime}$. So we have

Corollary. With assumptions of Lemma 2.2. the point spectrum of $\mathfrak{V}_{G}$ is symmetric

$$
\sigma\left(\mathfrak{V}_{G}\right)=\sigma\left(\mathfrak{V}_{G}\right)^{-1} .
$$

In some cases it occurs that there is an involution $J$ on $\mathscr{H}$ transforming $\mathfrak{A}$ onto $\mathfrak{A}^{\prime}$. This also implies, as was already pointed out by M. WINNINK $\left[2\right.$, IV.5, lemma], the symmetry of the point spectrum of $\mathfrak{V}_{G^{\prime}}$. Let us note that the following theorem is generally true

3.3. Theorem. Let $\mathfrak{V}_{\dot{t}^{*}}$ be a group of unitary operators acting on a Hilbert space $\mathscr{H}$. Assume there exists an involution $J$ commuting with $\mathfrak{V}_{G}$. Then the point spectrum of $\mathfrak{V}_{G}$ is symmetric and $J F(\chi) J=F\left(\chi^{-1}\right)$. Moreover, if $\mathfrak{V}_{G}$ is abelian, n-parameter and strongly continuous then $J F(\Delta) J=F(-\Delta)$ for each Borel set $\Delta \subset R^{n}$. 
We drope an easy proof of this theorem.

Under assumptions similar to those of Theorem 3.2. we can deduce also the symmetry and additivity of the continuous spectrum.

3.4. Theorem. Let $\mathfrak{V}_{G}$ and $\mathfrak{V}_{G_{1}}$ satisfy assumptions of Lemma 2.2. (with the same invariant vector $\Omega$ ). If $\mathfrak{V}_{G}$ is abelian, n-parameter and strongly continuous, so that we have

$$
V_{x}=\int \exp (i p x) F(d p), \quad x \in R^{n}
$$

then the spectrum of $V_{G}$ is symmetric

$$
\operatorname{supp} F(d p)=-\operatorname{supp} F(d p) .
$$

If, moreover, $\mathfrak{V}_{G_{1}}$ satisfies one of the equivalent conditions of Theorem 2.4., then the spectrum of $\mathfrak{V}_{G}$ is also additive

$$
\operatorname{supp} F(d p)+\operatorname{supp} F(d p) \subset \operatorname{supp} F(d p)
$$

provided $\lim _{x=0}\|A(x)-A\|=0$ on a dense $C^{*}$-algebra $\mathfrak{A}_{0} \subset \mathfrak{A}$.

Proof (it is a small modification of that in [10, Theorem $3 \mathrm{~b}]$ ). With $p_{1}, p_{2} \in \operatorname{supp} F(d p)$ and $\mathscr{N}_{1}, \mathscr{N}_{2}$-neighbourhoods of $p_{1}$ and $p_{2}$ respectively, there exist $A_{1}, A_{2} \in \mathfrak{A}_{0}$ different from zero and such that

$$
\operatorname{supp} \hat{A}_{i} \subset-\mathscr{N}_{i}
$$

Now, $\operatorname{supp} \hat{A}_{1}^{*}=-\operatorname{supp} \hat{A}_{1}$ and $A_{1}^{*} \Omega \neq 0$ since $\Omega$ is separating for A. Hence $F\left(-\mathscr{N}_{1}\right) A_{1}^{*} \Omega=A_{1}^{*} \Omega$ and we conclude that the spectrum is symmetric. To establish additivity we observe that $(*)$ also holds if we replace $A_{2}$ by $A_{2}(g), g \in G_{1}$ being arbitrary. But then

so that we have

$$
\operatorname{supp} \widehat{A_{1} A_{2}(g)} \subset-\left(N_{1}+N_{2}\right)
$$

$$
F\left(N_{1}+N_{2}\right) A_{1} A_{2}(g) \Omega=A_{1} A_{2}(g) \Omega
$$

and it is sufficient to show that $A_{1} A_{2}(g) \Omega \neq 0$ for some $g \in G_{1}$. Suppose, it is not so. Then $A_{1} V_{g} A_{2} A_{2}^{*}=0$ for all $g$ in $G_{1}$. Thus $A_{1} F_{01} A_{2} A_{2}^{*}=0$ and from Theorem 2.4. we infer that $\left(A_{2}^{*} \Omega, A_{2}^{*} \Omega\right) A_{1} \Omega=0$ contrary to the hypothesis. The proof is complete.

3.5. Theorem. Let $\mathfrak{V}_{G}$ and $\mathfrak{V}_{G_{1}}$ satisty assumptions of Lemma 2.2 (with the same invariant vector $\Omega$ ). Assume that $\mathfrak{B}$ is abelian and $\mathfrak{V}_{G_{1}} \cup \mathfrak{A}$ irreducible. Then the point spectrum of $\mathfrak{V}_{G}$ is a group and for each $\chi \in \sigma\left(\mathfrak{V}_{G}\right)$ there exist two unitary operators $\hat{\chi} \in \mathfrak{A}$ and $\check{\chi} \in \mathfrak{U}^{\prime}$ such that

$$
\begin{aligned}
V_{g} \tilde{\chi} & =\chi(g) \tilde{\chi} V_{g} \\
\tilde{\chi} F\left(\chi^{\prime}\right) \tilde{\chi}^{*} & =F\left(\chi \cdot \chi^{\prime}\right) \quad \text { for each } \quad \chi^{\prime} \in \sigma\left(\mathfrak{V}_{G}\right)
\end{aligned}
$$

where $\chi$ denotes either $\hat{\chi}$ or $\check{\chi}$.

Proof. Let $\chi \in \sigma\left(\mathfrak{V}_{G}\right)$. Then, as in Theorem 3.2., $F(\chi)$ is cyclic for $\mathfrak{A} \subset \mathfrak{R}$ and $\mathfrak{U}^{\prime} \subset \mathfrak{B}^{\prime}$. Hence $\mathfrak{Z}$ is isomorphic with $F(\chi) \mathfrak{B} F(\chi)$ and $\mathfrak{R}^{\prime}$ is isomorphic with $F^{\prime}(\chi) \mathfrak{R}^{\prime} F^{\prime}(\chi)$. Now, by the hypothesis and Corollary 2.3., $10^{*}$ 
$\mathfrak{P}^{\prime}$ is abelian and therefore there exists $\psi^{\prime} \in F(\chi) \mathscr{H}$ separating for $F(\chi) \mathfrak{R}^{\prime}$. Indeed, since $\Omega$ is separating for $\mathfrak{R}^{\prime}$, it follows that $\mathfrak{R}^{\prime}$ is countably decomposable, and by the isomorphism $\mathfrak{R}^{\prime} \cong F(\chi) \mathfrak{R}^{\prime}, F(\chi) \mathfrak{R}^{\prime}$ also does. The statement above follows from [7, Corollary, p. 20]. So, we find that $\psi^{\prime}$ is cyclic for $F(\chi) \Re F(\chi)=F(\chi) \mathfrak{B}$ or, equivalently, $\mathfrak{B} \psi^{\prime}$ is dense in $F(\chi) \mathscr{H}$. Since, on the other hand, $\mathfrak{B} \subset \mathfrak{R}$ and $\overline{\mathfrak{R} F(\chi) \mathscr{H}}$ $=\mathscr{H}$ it is clear that $\psi^{\prime}$ is cyclic for $\mathfrak{R}$ (and for $\mathfrak{A}$ also). Similarly, since $F(\chi) \mathfrak{R}^{\prime}=F(\chi) \mathfrak{Z}\left(2.3\right.$, Corollary), we infer that $\psi^{\prime}$ is also cyclic for $\mathfrak{B}^{\prime}$. Now, in view of the equality $\left[\Re \psi^{\prime}\right]=[\Re \Omega]$ we have $\left[\mathfrak{R}^{\prime} \psi^{\prime}\right] \sim\left[\mathfrak{R}^{\prime} \Omega\right]$ (see [7], Corollary, p. 232). That means there is a partial isometry $U \in \Re$ such that $U^{*} U=\left[\mathfrak{R}^{\prime} \Omega\right]$ and $U U^{*}=\left[\mathfrak{R}^{\prime} \psi^{\prime}\right]$. Observe at this point that $[\Omega] \leqq\left[\mathfrak{R}^{\prime} \Omega\right]=F_{0}$ and $\left[\psi^{\prime}\right] \leqq\left[\mathfrak{R}^{\prime} \psi^{\prime}\right]=F(\chi)$. Let us put $\psi=U \Omega$. Then $\psi \in\left[\mathfrak{Q}^{\prime} \psi^{\prime}\right]$ and is cyclic for $\mathfrak{A}$ and $\mathfrak{A}^{\prime}$. Furthermore, by Lemma 2.2 , for each $A$ in $\mathfrak{Q}^{\prime}$ we have

$$
\begin{aligned}
(\psi, A \psi) & =\left(\psi, \Phi^{\prime}(A) \psi\right)=\left(U \Omega, \Phi^{\prime}(A) U \Omega\right) \\
& =\left(\Omega, \Phi^{\prime}(A) U^{*} U \Omega\right)=\left(\Omega, \Phi^{\prime}(A) \Omega\right) \\
& =(\Omega, A \Omega) .
\end{aligned}
$$

In particular $\|A \psi\|=\|A \Omega\|$ for all $A$ in $\mathfrak{Q}^{\prime}$. Now, a linear operator $\hat{\chi}$ defined by $\hat{\chi} A \Omega=A \psi, A \in \mathfrak{A}^{\prime}$ is bounded on a dense set $\mathfrak{A}^{\prime} \Omega$. Moreover, with $A, B \in \mathfrak{U}^{\prime}$ we have

$$
(\hat{\tilde{\chi}} A \Omega, \hat{\chi} B \Omega)=(A \psi, B \psi)=(A \Omega, B \Omega)
$$

thus $\hat{\chi}$ is unitary, and by

$$
\hat{\chi} A B \Omega=A B \psi=A \hat{\chi} B \Omega
$$

it follows that $\hat{\chi}$ is in $\mathcal{A}$. With $g$ in $G$ we now have or

$$
V_{g} \hat{\chi} A \Omega=V_{g} A \psi=\chi(g) A(g) \psi=\chi(g) A(g) \Omega=\chi(g) \hat{\chi} V_{g} A \Omega
$$

$$
V_{g} \hat{\chi}=\chi(g) \hat{\chi} V_{g} .
$$

If also $\chi^{\prime}$ is in $\sigma\left(\mathfrak{V}_{G^{\prime}}\right)$ then by

it follows that

$$
\overline{\chi^{\prime}(g)} \hat{\chi} V_{g} \hat{\chi}^{*}=\overline{\chi^{\prime}(g)} \overline{\chi(g)} V_{g}
$$

$$
\hat{\chi} F\left(\chi^{\prime}\right) \hat{\chi}^{*}=F\left(\chi \cdot \chi^{\prime}\right) .
$$

The operators $\check{\chi}$ are constructed in much the same way.

Remark. Using the same method one can prove the following

Theorem. Let $\mathfrak{A}$ and $\mathfrak{V}_{G^{\prime}}$ be as in 2.1. Assume, there is a vector $\Omega$ cyclic for $\mathfrak{A}$ and invariant under $\mathfrak{V}_{G}$. Assume further that for each selfadjoint $A$ in $\mathfrak{A}$ we have

$$
\operatorname{conv}\{A(\hat{g})\}^{-} \cap \mathfrak{A}^{\prime} \neq \emptyset .
$$


Finally, assume that there is some other group $\mathfrak{V}_{G_{1}}$ commuting with $\mathfrak{V}_{G}$ and such that $\mathfrak{A}$ is invariant under $\mathfrak{V}_{G_{1}}$ and $\mathfrak{A} \cup \mathfrak{V}_{G_{1}}$ is irreducible. Then the point spectrum of $\mathfrak{V}_{G}$ is a group and for each $\chi \in \sigma\left(\mathfrak{V}_{G}\right)$ there exists a unitary operator $\check{\chi}$ in $\mathfrak{A}^{\prime}$ such that

$$
\begin{gathered}
V_{g} \check{\chi}=\chi(g) \check{\chi} V_{g}, \\
\check{\chi} F\left(\chi^{\prime}\right) \check{\chi}^{*}=F\left(\chi \cdot \chi^{\prime}\right) \text { for each } \chi^{\prime} \in \sigma\left(\mathfrak{V}_{G}\right) .
\end{gathered}
$$

It is easy to see that the proof follows from considerations given above, provided the mapping $\Phi$ is taken from [6, Theorem 3.1.].

\section{The Case of the KMS-Algebra}

If we consider the state of an infinite system in a thermal equilibrium with the temperature $(\beta k)^{-1}$, then in a natural way we can construct ([1], see also [2] where detailed analysis is given)

1. the Hilbert space $\mathscr{H}$,

2. the $C^{*}$-algebra $\mathfrak{A}_{0} \subset \mathfrak{L}(\mathscr{H})$,

3. the vector $\Omega$ cyclic for $\mathfrak{A}_{0}$ and for $\mathfrak{A}_{0}^{\prime}$,

4. the one-parameter, strongly continuous group of unitary operators $\left\{V_{t}\right\}$ such that

a) $A_{t}=V_{t} A V^{-1}$ is in $\mathfrak{A}_{0}$ for each $A \in \mathfrak{A}_{0}$ and $t \in \boldsymbol{R}$,

b) $\lim _{t=0}\left\|A_{t}-A\right\|=0$ for each $A \in \mathfrak{A}_{0}$,

c) $V_{t} \Omega=\Omega$ for all $t \in \boldsymbol{R}$

5. the involution $J$ such that $J A \Omega=T A * \Omega$ for each $A \in \mathfrak{A}=\left(\mathfrak{A}_{0}\right)^{\prime \prime}$, where $T=\exp (-\beta H / 2)$ and $V_{t}=\exp (i H t)$,

6 . The 3-parameter, strongly continuous group of unitary operators $\left\{U_{x}\right\}$ such that

a) $A_{x}=U_{x} A U_{x}^{-1}$ is in $\mathfrak{A}_{0}$ for each $A \in \mathfrak{A}_{0}$ and $x \in \boldsymbol{R}^{3}$,

b) $\lim _{x=0}\left\|A_{x}-A_{x}\right\|=0$ for each $A \in \mathfrak{A}_{0}$,

c) $U_{x} \Omega=\Omega$,

d) $U_{x}$ commute with $V_{t}$.

From the above one can derive the following conclusions [2]

i) the center $\mathbb{B}$ of $\mathfrak{A}$ is pointwise invariant with respect to time translations,

ii) for each $A$ in $\mathcal{B}$ we have $J A J=A^{*}$,

iii) $J \mathfrak{A} J=\mathfrak{A}^{\prime}$,

iv) $J \Omega=\Omega$.

Remark 1. It is easy to see that the existence of a conjugation $J$ satisfying 5 . is a necessary and sufficient condition for the state $\omega: A \rightarrow(\Omega, A \Omega)$ to satisfy the KMS boundary conditions (see [2]). These, on the other hand, are a consequence of the fact that the state $\omega$ is obtained as the limit of finite systems (described by the Grand Canoni- 
cal Ensemble). If we denote by $\omega_{v}$ the state of the system enclosed in a volume $V$, then it is a direct consequence of the properties of a trace that, $\omega_{v}(A B)=\omega_{v}\left(B A_{i \beta}\right)$ where $A_{i \beta}=(2 \pi)^{-1} \int \hat{A}(\varepsilon) e^{\beta \varepsilon} d \varepsilon$ and $\hat{A}(\varepsilon)$ $=\int e^{i \varepsilon t} A(t) d t$ has a compact support. It may be interesting that the converse statement is also true. Namely, one has the

Theorem. Let $\mathfrak{A}$ be an irreducible $C^{*}$-algebra acting on a Hilbert space $\mathscr{H}$. Let $\left\{V_{t}\right\}$ be a one-parameter, strongly continuous group of unitary operators such that for each $A$ in $\mathfrak{A}, A_{t}=V_{t} A V_{t}^{-1}$ is in $\mathfrak{A}$ and $\lim _{t=0}\left\|A_{t}-A\right\|=0$. Let $\omega$ be a normal state on $\mathfrak{A}$ such that $\omega(A B)$ $=\omega\left(B A_{i \beta}\right)$ and $\omega\left(A_{t}\right)=\omega(A)$. Then there exists $\varrho \in \mathcal{L}(\mathscr{H})$ with trace 1 , such that

and

$$
\omega(A)=\operatorname{Tr}(\varrho A)
$$

$$
\varrho=\frac{e^{-\beta H}}{\operatorname{Tr}\left(e^{-\beta H}\right)} .
$$

The sketch of the proof runs as follows. By a continuity requirement we have $\omega(A)=\operatorname{Tr}(\varrho A)$ with $\varrho \geqq 0$ and $\operatorname{Tr} \varrho=1$. By the invariance of $\omega$ under translations $A \rightarrow A_{t}$ we immediately have $V_{t} \varrho=\varrho V_{t}$. Now, since $\operatorname{Tr}(\varrho A B)=\operatorname{Tr}\left(\varrho B A_{i \beta}\right)=\operatorname{Tr}\left(A_{i \beta} \varrho B\right)$ for all $B$ in $\mathcal{E}(\mathscr{H})$, we conclude that $\varrho A=A_{i \beta} \varrho$ or

hence

$$
\varrho A=e^{-\beta H} A e^{\beta H} \varrho
$$

and therefore

$$
e^{\beta H} \varrho A=A e^{\beta H} \varrho \quad \text { for all } A \text { in } \mathcal{E}(\mathscr{H})
$$

$$
e^{\beta H} \varrho=\boldsymbol{I} .
$$

It follows from the above theorem that the Grand Canonical Ensemble is a unique state of a finite system satisfying KMS. But it does not means that all properties of a Gibbs ensemble which still hold in the limit $V \rightarrow \infty$ are contained in KMS. It is still possible that the Grand Canonical Ensemble has some additional properties, which follow from KMS for finite systems but not for infinite ones (and which possibly hold true in a thermodynamical limit). Such a possibility has been discussed in [12, Sec. IV].

Remark 2. In what follows the situation characterized by $1 .-6$. we denote as the "KMS-Algebra". These conditions are in particular satisfied in a representation for a finite system. In this case $\mathscr{H}$ is an algebra of Hilbert-Schmidt operators acting on a Fock space $\mathscr{H}_{F}(V)$. The question arises how one can distinguish between the KMS-Algebra of finite system and that of infinite one. It is easy to see that the case of finite system is ruled out by a requirement of irreducibility of $\mathfrak{A} \cup\left\{V_{t}\right\}$.

Remark 3. In [3] the properties of the spectrum of time and space translations has been investigated. In particular, the "energy" spectrum 
turnes out to be symmetric (with respect to the origin). What is an interpretation of this fact? It is not too difficult to check that for finite systems the spectrum of the "Hamiltonian" $H$ is identical with the set of all differences of eigenvalues of a "real" Hamiltonian in a Fock space $\mathscr{H}_{F}(V)$. One has to keep it in mind when dealing with infinite systems.

We will now show how the results of sec. 2 and sec. 3 can be applied to the case of the KMS-Algebra.

I. It is easy to see that the mapping $\Phi$ of Lemma 2.2. is uniquely given by

and

$$
\Phi(A) B \Omega=J T B^{*} F_{0} A^{*} \Omega \text { where } A, B \in \mathfrak{A}
$$

$$
\Phi^{\prime}(A) B \Omega=T J B^{*} F_{0} A^{*} \Omega \text { where } A, B \in \mathfrak{U}^{\prime} .
$$

Moreover if $F_{0} J=J F_{0}$ (particularly if $\mathfrak{V}_{G}$ commutes with time translations) then

$$
\Phi^{\prime}(J A J)=J \Phi(A) J \text { for all } A \in \mathfrak{A} .
$$

II. With notation of Corollary 2.3, let $G$ be a group of time translations and $G_{1}-$ a group of space translations. Then, if $F_{0}$ (resp. $E_{0}$ ) denotes a projection onto the subspace of all vectors invariant under time (resp. space) translations, we have

provided

$$
E_{0} \leqq F_{0} \text { and } \mathfrak{A} \cup\left\{V_{t}\right\} \subset\left(\mathfrak{A} \cup\left\{U_{x}\right\}\right)^{\prime \prime}
$$

$$
\mathscr{M}\left\{\left(\varphi,\left[U_{\hat{x}} A U_{\hat{x}^{-1}}, B\right] \psi\right)\right\}=0 \text { for all } A, B \in \mathfrak{A}, \quad \varphi, \psi \in E_{0} \mathscr{H} .
$$

This fact has been established by D. W. Robinson [3].

III. The spectrum of $\left\{V_{t}\right\}$ and $\left\{U_{x}\right\}$ is symmetric, moreover for any Borel set $\Delta$ we have

$$
J F(\Delta) J=F(-\Delta) \text { and } J E(\Delta) J=E(-\Delta)
$$

so that the point spectrum is also symmetric.

The above statement is a direct consequence of Theorem 3.3. It was exhibited in [3] and mentioned in [2, III. 1.].

IV. Assume that $\mathfrak{A} \cup\left\{U_{x}\right\}$ is irreducible. Then

a) the spectrum of $\left\{V_{t}\right\}$ and $\left\{U_{x}\right\}$ is additive (see 3.4);

b) the point spectrum of $\left\{U_{x}\right\}$ is a group and for each $p$ in $\sigma\left(U_{x}\right)$ there exist two unitary operators: $\hat{p} \in \mathfrak{U}$ and $\check{p} \in \mathfrak{U}^{\prime}$ such that (see 3.5)

$$
\begin{aligned}
& \qquad U_{x} \tilde{p}=e^{i p x} \tilde{p} U_{x}, \\
& \tilde{p} E(q) \tilde{p}^{*}=E(p+q) \text { if also } q \in \sigma\left(U_{x}\right), \\
& \text { where } \tilde{p} \text { denotes either } \hat{p} \text { or } \check{p} ; \\
& \text { c) for each } p \in \sigma\left(U_{x}\right) \text { we have } E(p) \leqq F_{0} .
\end{aligned}
$$


To obtain the last relation, observe that by the definition of $\hat{p}$ and since $E(p)=\hat{p} E_{0} \hat{p}^{*}$ is one-dimentional, we have

$$
1=\|\Omega\|=\left\|J \hat{p}^{*} \Omega\right\|=\|T \hat{p} \Omega\|=\exp [-\varepsilon(p) \beta / 2]\|\hat{p} \Omega\|=e^{-\varepsilon(p) \beta / 2}
$$

what is possible only if $\varepsilon(p)=0$.

It is easy to see that $c$. implies that $\hat{p}$ and $\check{p}$ commute with time translations and therefore

$$
J \hat{p} J=\check{p}^{*} .
$$

If one applies the above to time translations, then one obtains:

V. If $\mathfrak{A} \cup\left\{V_{t}\right\}$ is irreducible then $\Omega$ is the only eigenstate of $\left\{V_{t}\right\}$.

The above result is a generalization of [2, p. 46, Lemma 2$]$.

VI. Under assumptions of IV. let $p \in \sigma\left(U_{x}\right)$. Then the operator $J_{p}=\check{p} J_{p}^{*}$ is an involution, and if $\psi_{p}=\hat{p} \Omega$ then for each $A$ in $\mathfrak{A}$ we have

$$
\begin{aligned}
T A \psi_{p}=T A \hat{p} \Omega & =J \hat{p}^{*} A^{*} \Omega=J \hat{p}^{*} A^{*} \check{p} * \psi_{p} \\
& =\check{p} J \check{p}^{*} A^{*} \psi_{p}=J_{p} A^{*} \psi_{p},
\end{aligned}
$$

so that we have some kind of a degeneracy: the vector $\psi_{p}$ and the involution $J_{p}$ have the same property as $\Omega$ and $J$.

VII. The question arises are the operators $\hat{p}$ in the center of $\mathfrak{A}$. It is easy to see that the answer is "yes" if $\mathfrak{A}$ is $t$-asymptotically abelian i.e. $\mathscr{M}\left\{\left(\varphi,\left[V_{\hat{t}} A V_{\hat{t}}^{-1}, B\right] \psi\right)\right\}=0$ for all $A, B \in \mathfrak{I}$ and $\varphi, \psi \in F_{0} \mathscr{H}$.

If it is so, then by 3.5 (applied to time translations) and by the considerations analogous to those in VI. it follows that zero is the only eigenvalue of the "Hamiltonian" $H$. This may be also expected from the interpretation of the spectrum of $H$ (see Remark 3 of this section). The last statement is a further generalization of $V$.

Acknouledgements. The author wishes to thank Professor J. T. Łopuszański for his interest and reading the manuscript.

\section{References}

1. HaAg, R., N. M. Hugenholtz, and M. Winnink: Commun. Math. Phys. ๘, 215 (1967).

2. WINNINk, M.: An application of $\mathrm{C}^{*}$-algebras to quantum statistical mechanics of systems in equilibrium. Thesis, Groningen 1968.

3. Robinson, D. W.: Commun. Math. Phys. 7, 337 (1968).

4. Kovacs, I., and J. Szücs: Acta Sc. Math. 27, 233 (1966).

4a. Dopltcher, S., D. Kastler, and E. Stormer: Invariant states and Asymptotic Abelianness, preprint 1968.

5. - - Commun. Math. Phys. 7, 1 (1968). 
6. Stormer, E.: Commun. Math. Phys. 5, 1 (1967).

7. Dixmier, J.: Les algebres d'opérateurs dans l'espace Hilbertien. Paris 1957.

8. Araki, H., and H. Miyata: On KMS Boundary Condition, preprint 1968.

9. Doplicher, S., R. W. Kadison, D. Kastler, and D. W. Robinson: Commun. Math. Phys. 6, J0I (1967).

10. Robinson, D. W., and D. Ruelle: Ann. Inst. Henri Poincaré Vol. VI, n ${ }^{0} 4$, 299 (1967).

11a. JADCZYK, A. Z.: Commun. Math. Phys. 12, 58 (1969).

11b. JADCZYK, A. Z., and L. NIKolova: Internal symmetries and observables, preprint (1969).

12. Hugenholtz, N. M., and J. D. Wieringa: On locally normal states in quantum statistical mechanics, preprint (1968).

A. Z. JADCZYK

Institute of Theoretical Physics

University of Wroclaw

Cybulskiego 36

Wroclaw, Poland 